Kansas State University Libraries

New Prairie Press

\title{
NONLINEAR MIXED MODELS TO EVALUATE EFFECTS OF ENVIRONMENT CONDITIONS, HAIR COAT, AND ANCHOR LENGTH ON BODY TEMPERATURE DURING AFTERNOON MILKING OF HOLSTEIN COWS IN HAWAII
}

N Aitha
A. M. Parkhurst
C. N. Lee
P. E. Hillman

See next page for additional authors

Follow this and additional works at: https://newprairiepress.org/agstatconference

Part of the Agriculture Commons, and the Applied Statistics Commons

\section{c) (1) $\odot$}

This work is licensed under a Creative Commons Attribution-Noncommercial-No Derivative Works 4.0 License.

\section{Recommended Citation}

Aitha, N; Parkhurst, A. M.; Lee, C. N.; and Hillman, P. E. (2008). "NONLINEAR MIXED MODELS TO EVALUATE EFFECTS OF ENVIRONMENT CONDITIONS, HAIR COAT, AND ANCHOR LENGTH ON BODY TEMPERATURE DURING AFTERNOON MILKING OF HOLSTEIN COWS IN HAWAII," Conference on Applied Statistics in Agriculture. https://doi.org/10.4148/2475-7772.1102

This is brought to you for free and open access by the Conferences at New Prairie Press. It has been accepted for inclusion in Conference on Applied Statistics in Agriculture by an authorized administrator of New Prairie Press. For more information, please contact cads@k-state.edu. 
Author Information

N Aitha, A. M. Parkhurst, C. N. Lee, and P. E. Hillman

This is available at New Prairie Press: https://newprairiepress.org/agstatconference/2008/proceedings/11 


\title{
NONLINEAR MIXED MODELS TO EVALUATE EFFECTS OF ENVIRONMENT CONDITIONS, HAIR COAT, AND ANCHOR LENGTH ON BODY TEMPERATURE DURING AFTERNOON MILKING OF HOLSTEIN COWS IN HAWAII
}

\author{
N Aitha ${ }^{1}$, A. M. Parkhurst ${ }^{1}$, C. N. Lee ${ }^{2}$ and P. E. Hillman ${ }^{3}$ \\ 1. Department of Statistics, University of Nebraska at Lincoln \\ 2. Department of Human Nutrition, Food and Animal Sciences, University of Hawaii- \\ Manoa \\ 3. Biological and Environmental Engineering Department, Cornell University
}

\begin{abstract}
A hot thermal environment is one factor that causes loss of activity, productivity and even death in cows. One reason may be the effect of hair coat (black or white) on body temperature during different activities and environment conditions. In this study, we attempt to characterize the relationship between body temperature over time using activity, physiological and environmental effects with a nonlinear regression model. The fixed and mixed versions of models are examined. We also examine the effect of the measuring device, anchor length (long or short). Environmental effects, such as, air temperature, thermal heat index (THI), relative humidity, solar radiation, and wind speed, For PM Milking activity, differences in hair coat and anchor length tested as treatment effects. We present a detailed approach focusing on the rate of change in animal body temperature using the nonlinear regression model and investigate how the dynamics between the random-effects changes.
\end{abstract}

\section{INTRODUCTION}

Higher body temperature and hot environment conditions influence the health and productivity of an animal. Heat stress affects animals' growth and behavior during daily activity periods; and, results in decreased feed intake. Heat stress causes economic losses to livestock producers. In dairy operations, summer depression in milk production can be as high as $25 \%$. In extreme cases, heat stress can even result in death of vulnerable animals. Events like the heat waves in Iowa (1995) and Nebraska (1999) resulted in the death loss of more than 3,500 head of cattle and millions of dollars to the beef industry. The July 2006 heat wave in California resulted in over \$1 billion losses and higher food prices due to a lower production.

A unique feature of the study is the site of the Mt Kohala dairy. Mt Kohala is located on the northern corner of the Big Island of Hawaii. The dairy is a pasture based operation that provides a unique situation to study cow behavior and physiology in a natural environment. Many dairy producers use fans to cool cows in the summer (Baek, et. al., 2001). This region has wind speeds that constantly exceed $10 \mathrm{mph}$ providing a natural cooling system. In addition, the region has sub-tropical climate with high solar intensity and high air temperature. 
Since decrease in milk production is a primary concern, we focus on milking activities. There are two milking activities at the dairy: am (0500h) and pm (1700h). The pm milking is used in the study because it is the most practical. It is difficult to observe cows at $0500 \mathrm{~h}$ as it is still dark. Moreover, we expect the cows to be cool throughout the night prior to the am milking; hence, they are not thermally stressed from elements of weather.

Coat color is one characteristic of vulnerable animals. The coat color associated with different hair colors may be one of the factors that cause differences in response to a hot environment. Animals with dark color hair are thought to be more heat stressed than light-color animals due to the adsorption of solar radiation. Previous studies from Hawaii (Lee, et.al, 2003) and from the Virgin Islands (Godfrey and Hansen, 1996) show that there are more black cows in a dairy herd in the tropics. Further investigations suggested that these animals had higher dorsal skin temperature facilitating higher sweating rates (Hillman, et. al.,2001), (Geebremedhin,et.al,2007). Since black cows absorbed more heat and solar radiation load, it would be informative to compare their behavior to that of white cows in natural setting with thermally challenging conditions.

A secondary issue in this experiment is the length of the anchors on the temperature probes. In previous unpublished work, we modified the short anchor to longer projections/fingers so as to prevent the cows from expelling the instrument out of the vagina. We did notice that the long anchor held the temperature probe inline with the anterior-posterior position of the cow. The direction of short anchor temperature probe rotated inside the cow. Thus, we examine the effect of anchor length.

There are three objectives for this study. First, model the changes in body temperature over time for the activity period, afternoon milking. Second, identify environment effects that influence changes in body temperature and include the most important environmental variable as a second covariate. Third, test for differences in hair color and anchor length of measuring instrument in the presence of mixed effects and an environmental covariate.

This paper is organized as follows: In Section 2, we describe the experimental design of the data, the statistical models, model building techniques, and diagnostics. In Section 3, we identify the significant random effects; examine the environmental covariates; discuss the treatment effects and the diagnostics for the final model. In Section 4, we present our conclusions. Finally, in Section 5, we summarize the study.

\section{METHODS AND MATERIALS}

\section{2.a Experimental Design}

A study of ten Holstein cows was conducted by Drs. Chen Lee and Peter Hilman at Mt. Kohala in Hawaii from Aug $8^{\text {th }} 2006$ to Aug $9^{\text {th }} 2006$. Five Holstein cows were shaded as eighty percent black and the other five Holstein cows were eighty percent white. Five Holstein cows were measured using the short anchor probe and the other five Holstein cows were measured using long anchor probe. The day was divided into Activity 
periods. The times for milking and feeding activities are given in the Table 1 and Fig 1 . For this paper we focus on the pm milking. Body temperature $(\mathrm{Tb})$ was recorded every minute (as fraction of hr) for up to two days for each activity period as was the environmental variables: Air temperature (Ta), solar radiation, relative humidity $(\mathrm{RH})$, wind speed and thermal heat index (THI).

\begin{tabular}{|c|c|}
\hline \multicolumn{2}{|c|}{ Table 1. Activity Periods } \\
\hline Activity & Time \\
\hline MilkAM & 5:00am to $7: 30 \mathrm{am}$ \\
\hline FeedAM & $8: 00 \mathrm{am}$ to $9: 15 \mathrm{am}$ \\
\hline FeedPM & $15: 00 \mathrm{pm}$ to $16: 45 \mathrm{pm}$ \\
\hline MilkPM & $17: 00 \mathrm{pm}$ to $18: 45 \mathrm{pm}$ \\
\hline
\end{tabular}

The experimental design for $\mathrm{Tb}$ in each activity period is a completely random design with repeated measures over time and environmental covariates with a 2 x 2 factorial treatment design. The two factors are hair color and anchor length.

Fig 1. Observed Body temperature versus Time for cow 3238

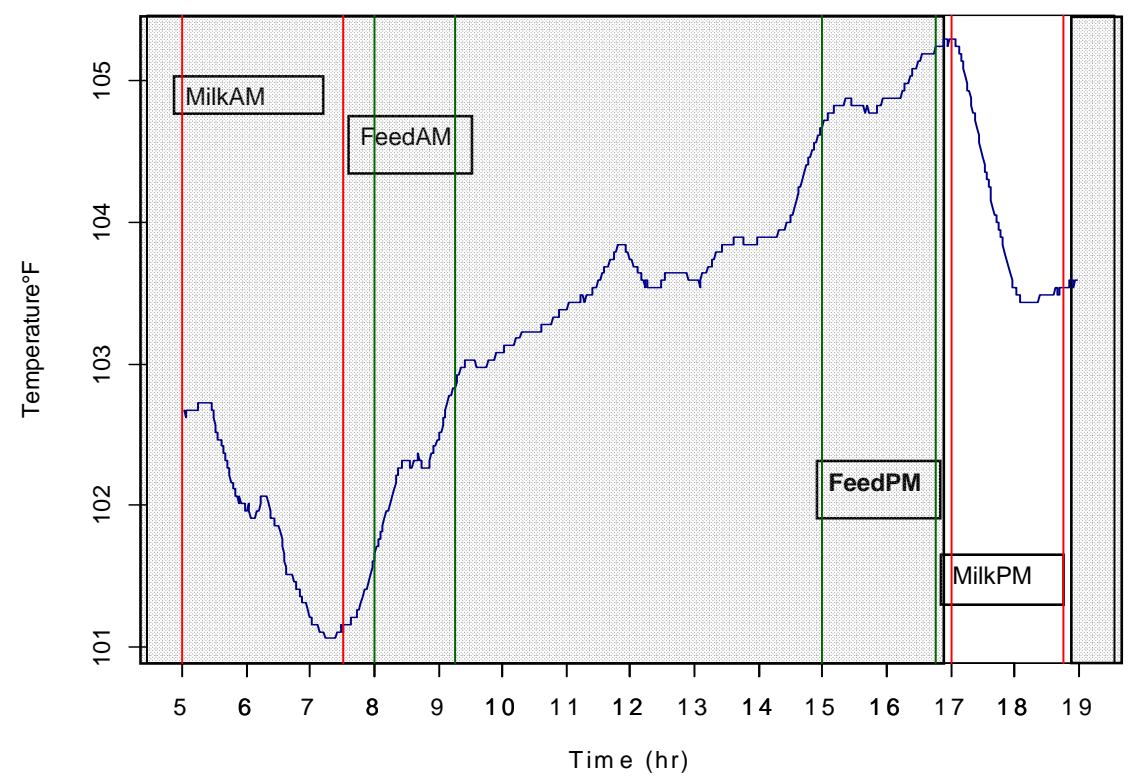

\section{2.b. Statistical Models}

When choosing a regression model to characterize how the observed body temperature varies with time, we considered both linear and nonlinear models. We chose the exponential regression as the nonlinear model in part because a similar study, by Huang and Parkhurst (2006) showed models based on the exponential regression were superior to the linear models. Moreover, the nonlinear exponential regression has a realistic interpretation. It is derived from the differential equation which is based on the assumption that the rate of change in $\mathrm{Y}$, the observed body temperature, is proportional to the increase in $\mathrm{Y}$ above the animal's baseline body temperature.

$$
\partial \mathrm{Y} / \partial \mathrm{X}=\mathrm{kappa}^{*}(\mathrm{Y}-\mathrm{delta})
$$


The differential equation leads to the asymptotic (exponential) regression,

$$
\mathrm{Y}=\text { delta }+ \text { beta } * \mathrm{e}^{- \text {kappa*X}^{*}}
$$

Where $\mathrm{Y}$ is the observed body temperature, $\mathrm{X}$ is time (in hours), delta is minimum (baseline) body temperature, beta is a range parameter, which represents the difference between maximum and baseline body temperature, and kappa is the rate parameter.

We compared both linear and nonlinear models and found the nonlinear regression models provide the best fit, give more precision in treatment differences and offer more interpretable parameters.

\section{2.b.i. Nonlinear Mixed Model}

In our study, both cow and day are crossed with each other. The NLME function in R is very powerful for fitting nonlinear mixed models with nested random effects, but it does not fit nonlinear mixed effects with crossed random effects. Zhou and Parkhurst (2006) investigated different computer programs for fitting nonlinear mixed-effect models. They developed a method that enables NLME in R to fit a nonlinear mixed-effects model with crossed random effects based on Goldstein's idea for the linear mixed-effects model (Goldstein, 1999). We implemented their method. We defined a variable called event, a combination of cow and day. Then we grouped the observations by event, the lowest hierarchical grouping factor.

The model is defined as:

$$
\begin{gathered}
\mathbf{Y}_{\mathbf{i}}=\text { delta }+ \text { beta }^{*} \mathbf{e}^{\left(- \text {kappa }^{*} \mathrm{X}\right)}+\boldsymbol{\varepsilon}_{\mathbf{i}} \\
{\left[\begin{array}{l}
\text { delta } \\
\text { beta } \\
\text { kappa }
\end{array}\right]=\left[\begin{array}{l}
\boldsymbol{\delta}+\mathbf{b}_{\delta} \\
\boldsymbol{\beta}+\mathbf{b}_{\beta} \\
\boldsymbol{\kappa}+\mathbf{b}_{\boldsymbol{\kappa}}
\end{array}\right] \quad \mathbf{b}=\left[\begin{array}{l}
\mathbf{b}_{\delta} \\
\mathbf{b}_{\beta} \\
\mathbf{b}_{\boldsymbol{\kappa}}
\end{array}\right] \quad \psi=\left[\begin{array}{ccc}
\sigma_{\text {delta }}^{2} & 0 & 0 \\
& \sigma_{\text {beta }}^{2} & 0 \\
& \sigma^{2}{ }_{\text {kappa }}
\end{array}\right]}
\end{gathered}
$$

Where, $\mathbf{b} \sim \mathbf{N}(\mathbf{0}, \boldsymbol{\psi})$ and $\boldsymbol{\varepsilon} \sim \mathbf{N}\left(\mathbf{0}, \boldsymbol{\sigma}^{2} I\right)$ are both independent of each other. Both fixed and random effects are considered for delta, beta, and kappa. The fixed effects, $\delta, \beta$, and $\mathrm{K}$ represent population means. Random effects $b_{\delta}, b_{\beta}, b_{K}$ represent the deviation from the population means associated with $i^{\text {th }}$ Event $(i=1,2, . .20) . \quad Y_{i}$ represents the body temperature for $\mathrm{i}^{\text {th }}$ event and $\mathrm{X}$ represents the time in hours.

\section{2.b.ii Inclusion of Covariate Effect}

After identify the significant random-effects, we include an environmental response as a covariate in the model Eq $1 \quad \mathbf{Y}_{\mathbf{i}}=$ delta + beta $* \mathrm{e}^{\left(- \text {kappa }^{*} \mathrm{X}\right)}+\varepsilon_{\mathrm{i}}$

$$
\left[\begin{array}{l}
\text { delta } \\
\text { beta } \\
\text { kappa }
\end{array}\right]=\left[\begin{array}{l}
\left(\boldsymbol{\delta}+b_{\delta}\right)+\gamma_{0} X_{T a} \\
\left(\boldsymbol{\beta}+b_{\beta}\right)+\gamma_{0} X_{T a} \\
\left(\kappa+b_{\kappa}\right)+\gamma_{0} X_{T a}
\end{array}\right] \quad \mathrm{b}=\left[\begin{array}{l}
b_{\delta} \\
b_{\beta} \\
b_{\kappa}
\end{array}\right]
$$


Both fixed and random effects were depicted as in Eq 1.The parameter $\gamma_{0}$ represents the slope of the air temperature effect and $\mathrm{X}_{\mathrm{Ta}}$ is the air temperature response $(\mathrm{F})$. Assumptions are the same as described in Eq1.

\section{2.b.iii Inclusion of Treatment Effects}

After identify covariate effects, we include treatment factors in model Eq 2

$$
\begin{aligned}
& Y_{i}=\text { delta }+ \text { beta }^{*} e^{(-k a p p a * X)}+\varepsilon_{i} \\
& {\left[\begin{array}{l}
\text { delta } \\
\text { beta } \\
\text { kappa }
\end{array}\right]=\left[\begin{array}{l}
\left(\delta+\alpha_{1} C_{k}+\alpha_{2} A_{\ell}+\alpha_{3} C A_{k \ell}\right)+\left(\gamma_{0}+\gamma_{1} C_{k}+\gamma_{2} A_{\ell}+\gamma_{3} C A_{k \ell}\right) X_{T a}+b_{\delta} \\
\left(\beta+\alpha_{1} C_{k}+\alpha_{2} A_{\ell}+\alpha_{3} C A_{k \ell}\right)+\left(\gamma_{0}+\gamma_{1} C_{k}+\gamma_{2} A_{\ell}+\gamma_{3} C A_{k \ell}\right) X_{T a}+b_{\beta} \\
\left(\kappa+\alpha_{1} C_{k}+\alpha_{2} A_{\ell}+\alpha_{3} C A_{k \ell}\right)+\left(\gamma_{0}+\gamma_{1} C_{k}+\gamma_{2} A_{\ell}+\gamma_{3} C A_{k \ell}\right) X_{T a}+b_{\kappa}
\end{array}\right] \quad b=\left[\begin{array}{l}
b_{\delta} \\
b_{\beta} \\
b_{\kappa}
\end{array}\right]} \\
& C_{k}=\left\{\begin{array}{ll}
0, \text { color type } & \mathrm{k}=\text { black } \\
1, \text { color type } & \mathrm{k}=\text { white }
\end{array} \quad \mathrm{A}_{\ell}=\left\{\begin{array}{l}
0, \text { anchor length } \quad \ell=\text { long } \\
1, \text { anchor length } \quad \ell=\text { short }
\end{array}\right.\right.
\end{aligned}
$$

Where $\alpha_{1}$ and $\alpha_{2}$ represent main effects of color and anchor, while $\alpha_{3}$ represents the coloranchor interactions. The covariate effects of air temperature are $\gamma_{0}$ for the intercept and $\gamma_{1}, \gamma_{2}, \gamma_{3}$ for the slope of color, anchor length and color-anchor interaction, respectively. Assumptions are as stated in Eq1.

\section{2. c Model Building and Analysis}

\section{2. c.i Random Effects Specification}

We begin with Eq 1, the nonlinear mixed effects model with no covariate and no treatments. To examine the random-effects variation, within-event errors, $\varepsilon_{i}$, are initially assumed to be independent $\mathrm{N}\left(0, \sigma^{2} \mathrm{I}\right)$ and all three parameters are initially considered to be mixed with a fixed population mean and random-effects for event. To avoid convergence problems with the optimization algorithm used in NLME (Pinheiro and Bates, 2000), a diagonal structure is assumed for the between event random-effects variance-covariance matrices. To identify which parameters, if any, require random effects, we fit Eq 1 for several sets of diagonal random-effects structures and check for model equivalency. The random-effects are removed one parameter at a time to produce a reduced model. The reduced model is compared to the full model with all random-effects on the diagonal. If the likelihood ratio test (LRT) is not significant, then the removal of random-effect is justified. In this way, we obtain the significant random effects for the model under the assumptions of diagonal variance-covariance matrices of random effects and independence of error with no covariate and no treatment effects. 


\section{2.c. ii Inclusion of Covariate Effect}

After identifying the significant random-effects, we examine each environmental response as a covariate in a nonlinear mixed effects model, Eq 2. First, air temperature is defined as covariate and the model with the covariate is obtained. We then use the LRT (between the model with and without covariate) to see if the covariate improves model performance. We repeat this process for each of the other environmental variables (THI, $\mathrm{RH}$, solar and wind speed). We use the information criteria (smallest AIC, BIC) to compare the environmental effects. In this way, we identify the single most important environment effect that influence changes in body temperature.

\section{2.c. iii Inclusion of Treatment Effects}

After identifying the significant random-effects and environmental covariate, we include the $2 \times 2$ factorial treatment design for color and anchor length in the nonlinear mixed effects model, Eq 3. To determine the most parsimonious model the highest order fixedeffects with large p-values are removed one at a time. Each time we check model performance with the LRT (between the model with and without the fixed-effect). If the LRT is non-significant, the fixed-effect is removed. In this way, a model is obtained including all significant highest order fixed-effects.

\section{2. c.iv Model Diagnostics}

After the random effects, covariate and fixed-effects are specified, we examine the residuals, random-effects, and nonlinear behavior. For the within-event errors, the normality assumption is investigated by the normal quantile plot of the standardized residuals and the Shapiro-Wilks test. A scatter plot of standardized residuals versus fitted values is used to investigate departure from the homogeneity and independent assumptions. The random-effects are checked for correlation among parameters with matrix of scatter plots of random-effects. The Box plot of residuals by event shows how the random-effects vary about zero.

Two additional assumptions are needed for least squares estimation of parameters in nonlinear regression: planarity and uniformity of coordinates. There are several ways to assess how close a model is to satisfying these asymptotic properties, also called close-tolinear behavior (Ratkowsky 1983, section 1.4). Bates and Watts $(1980,1988)$ proposed relative measures for intrinsic and parameter-effects curvature measures.

Intrinsic curvature (IN) measures the relative curvature of the expectation surface at the point of convergence. In linear regression, the expectation surface is a plane and IN always equals zero. For nonlinear regression, IN increases as the curvatures of the expectation surface increases, invalidating the estimation procedure. Large IN values indicate unacceptable deviation from the tangent plane assumption. A reference value of 0.4 indicates the relative deviation of the expectation surface from tangent plane relative as a percentage of the radius of the confidence disk is $21 \%$ 
Parameter-effects curvature, PE, measures the lack of uniformity of the parameter lines on the tangent plane. In linear regression, the parameter contours are parallel and equally-spaced when projected onto the tangent plane. Again the reference value is 0.4. Addition measures of close-to-linear behavior for the parameters and reference values are: percent bias $(<0.1)$, percent excess variance $(\lambda=0.1)$ and skewness.

Another way to assess nonlinear behavior is to observe the contour (profile) plots. A contour plot is a graphical technique for displaying the likelihood contours of parameter estimates. If the parameter contour is close to elliptical then the parameter estimates have close-to-linear behavior.

A final assessment of the adequacy of the nonlinear mixed-effects model is given by a plot of the augmented predictions. This plot is used to show the population predictions (obtained by random effects by setting zero) and within-group predictions (estimated random effects) are displayed in the same plot. Population prediction and within-group predictions are close to observed values then mixed model fits the data well.

\section{Results and Discussion}

\section{3. i. Selection of Random Effects}

Model 1 uses the full diagonal random-effects structure specified in Eq 1. The estimated standard deviations are given in Table 2. The random-effects are removed one parameter at a time to produce the reduced two random-effects Models 2-4. Table 2 shows the standard deviations of the random-effects and within events residual. The residuals range from 0.1144 to 0.1668 . The full Model 1 has the smallest residual suggesting all randomeffects are needed.

Table 2. Standard deviations of Random-effects and Residual for Nonlinear Mixed Models: Milk PM Activity

\begin{tabular}{|c|c|c|c|c|c|}
\hline \multirow{2}{*}{ Random Effect } & \multirow{2}{*}{} & \multicolumn{3}{|c|}{ Non-Linear Mixed Models } & \multirow{2}{*}{ Residual } \\
\cline { 3 - 5 } & Model & Delta & Beta & Kappa & \\
\cline { 3 - 5 } & 1 & $\mathbf{0 . 7 1 6 3 3}$ & $\mathbf{0 . 7 0 5 8 2}$ & $\mathbf{1 . 6 6 9 1}$ & $\mathbf{0 . 1 1 4 4}$ \\
\hline $\begin{array}{c}\text { Delta, Beta, } \\
\text { Kappa }\end{array}$ & & & & & \\
\hline Delta, Beta & 2 & 0.68503 & 0.6428 & - & 0.1381 \\
\hline Beta, Kappa & 3 & - & 1.0211 & 0.8444 & 0.1613 \\
\hline Delta, Kappa & 4 & 0.8210 & - & 17.942 & 0.1668 \\
\hline
\end{tabular}

The likelihood ratios and information criteria are used to compare the models (Table 3). The likelihood ratio tests are significant ( $\mathrm{p}$-value $<0.0001$ ) when we compare Model 1 vs. 2, 3 and 4. Model 1 has the smallest AIC, BIC and largest log-likelihood indicating Model 1 with random-effects for delta, beta and kappa explains more of variability than the other models. Thus, we use Model 1 for further analysis. 


\begin{tabular}{|c|c|c|c|c|c|c|c|}
\hline $\begin{array}{l}\text { Random } \\
\text { Effect }\end{array}$ & Model & $\overline{\mathrm{AIC}}$ & $\mathrm{BIC}$ & $\begin{array}{c}\text { Log- } \\
\text { Likelihood }\end{array}$ & Compare & LRT & $\overline{p \text {-value }}$ \\
\hline $\begin{array}{c}\text { Delta, Beta, } \\
\text { Kappa }\end{array}$ & 1 & -2782.3 & -2742.7 & 1398.159 & & & \\
\hline Delta, Beta & 2 & -2085.3 & -2052.4 & 1048.674 & 1 vs 2 & 698.9 & $<0.0001$ \\
\hline Beta, Kappa & 3 & -1427.4 & -1393.5 & 719.7468 & 1 vs 3 & 1356.8 & $<0.0001$ \\
\hline Delta, Kappa & 4 & -1245.7 & -1211.8 & 628.8746 & 1 vs 4 & 1538.5 & $<0.0001$ \\
\hline
\end{tabular}

\section{3. ii. Selection of Environmental Covariate}

After identifying the significant random-effects, each environmental variable is included in Model 1, one at a time, Models for air temperature (Ta), thermal heat index (THI), relative humidity $(\mathrm{RH})$, solar radiation (Solar), and, wind speed (Wind) are numbered 59. Model 1 is nested within Models 5-9, so likelihood ratio test is used to check if the inclusion of covariate effects causes a significant change in model performance. The likelihood ratio tests are significant $(p-v a l u e<0.0001)$ for each covariate when we compare the model 5-9 with Model 1 (Table 4). Each environmental variable significantly improves Model 1 . Next we find the single most important environmental variable. The Models 5-9 are non-nested models so we find the best model by looking at the AIC, BIC and log-likelihood values. Information criteria, log likelihood and residual all point to Model 5 suggesting Ta, air temperature, is the most important environment effect needed to reduce the variability associated with environmental variables.

\section{Table 4. Information Criterion, Log-likelihoods and Likelihood Ratio Test for Environment Covariates: Milk PM Activity}

\begin{tabular}{|l|l|l|l|l|l|l|l|l|l|}
\hline $\begin{array}{l}\text { Random } \\
\text { Effects }\end{array}$ & $\begin{array}{l}\text { Mo } \\
\text { del }\end{array}$ & $\begin{array}{l}\text { Covariat } \\
\text { e Effect }\end{array}$ & AIC & BIC & $\begin{array}{l}\text { Log- } \\
\text { Likeliho } \\
\text { od }\end{array}$ & $\begin{array}{l}\text { Residu } \\
\text { al }\end{array}$ & $\begin{array}{l}\text { Compa } \\
\text { re }\end{array}$ & LRT* & p-value \\
\hline $\begin{array}{l}\text { Delta, Beta, } \\
\text { Kappa }\end{array}$ & 1 & None & -2782.32 & -2742.77 & 1398.159 & 0.1144 & & & \\
\hline $\begin{array}{l}\text { Delta, Beta, } \\
\text { Kappa }\end{array}$ & 5 & Ta & -4317.24 & -4260.75 & 2168.622 & 0.0782 & 5 vs 1 & 1540.927 & $<0.0001$ \\
\hline $\begin{array}{l}\text { Delta, Beta, } \\
\text { Kappa }\end{array}$ & 6 & THI & -4179.57 & -4123.07 & 2099.785 & 0.0808 & 6 vs 1 & 1403.251 & $<0.0001$ \\
\hline $\begin{array}{l}\text { Delta, Beta, } \\
\text { Kappa }\end{array}$ & 7 & RH & -3714.74 & -3658.24 & 1867.368 & 0.0906 & 7 vs 1 & 938.418 & $<0.0001$ \\
\hline $\begin{array}{l}\text { Delta, Beta, } \\
\text { Kappa }\end{array}$ & 8 & Solar & -3299.24 & -3242.75 & 1659.621 & 0.1005 & 8 vs 1 & 522.9251 & $<0.0001$ \\
\hline $\begin{array}{l}\text { Delta, Beta, } \\
\text { Kappa }\end{array}$ & 9 & Wind & -2908.15 & -2851.66 & 1464.079 & 0.1102 & 9 vs 1 & 131.8403 & $<0.0001$ \\
\hline
\end{tabular}




\section{3. iii. Inclusion of Treatment effects}

After the identifying the random-effects and environmental covariate effect, we incorporate the $2 \times 2$ factorial treatment design to examine the effects of hair coat color and anchor length on each parameter - Model 10 (Table 5). We found that all parameters (delta, beta, and kappa) have a significant interaction between color, anchor length and Ta slope, (p-value $<0.05)$.

Table 5. Estimates of Fixed Effect Coefficients for Color and Anchor Length Model 10: Milk PM Activity

\begin{tabular}{|c|c|c|c|c|}
\hline Parameter & Fixed Effects & Estimate & $\begin{array}{l}\text { Std. } \\
\text { Error }\end{array}$ & P-value \\
\hline \multirow[t]{8}{*}{ Delta } & Intercept & 108.9259 & 0.677759 & 0 \\
\hline & Color: (W) & 39.73241 & 6.738491 & 0 \\
\hline & Anchor: $(\mathrm{S})$ & 1.88378 & 1.513276 & 0.2133 \\
\hline & Temp: $(\mathrm{T})$ & -0.08942 & 0.008245 & 0 \\
\hline & Color X anchor (WS) & -33.1423 & 6.948582 & 0 \\
\hline & Color X Temp (WT) & -0.54848 & 0.091029 & 0 \\
\hline & Anchor X Temp (ST) & -0.02301 & 0.019226 & 0.2316 \\
\hline & $\begin{array}{l}\text { Color X Anchor X } \\
\text { Temp(WST ) }\end{array}$ & 0.4505 & 0.09359 & 0.000 \\
\hline \multirow[t]{8}{*}{ Beta } & Intercept & 17.03525 & 3.230332 & 0 \\
\hline & Color: (W) & -55.1849 & 8.071053 & 0 \\
\hline & Anchor: (S) & 18.5226 & 6.751511 & 0.0061 \\
\hline & Temp: (T) & -0.20149 & 0.041106 & 0 \\
\hline & Color X anchor (WS) & 37.67052 & 10.39829 & 0.0003 \\
\hline & Color X Temp (WT) & 0.73118 & 0.107585 & 0 \\
\hline & Anchor X Temp (ST) & -0.23282 & 0.086147 & 0.0069 \\
\hline & $\begin{array}{l}\text { Color X Anchor X } \\
\text { Temp(WST) }\end{array}$ & -0.50716 & 0.136327 & 0.0002 \\
\hline \multirow[t]{8}{*}{ Kappa } & Intercept & 51.30672 & 4.226634 & 0 \\
\hline & Color: (W) & -43.7246 & 4.553175 & 0 \\
\hline & Anchor: (S) & 23.56734 & 7.290801 & 0.0012 \\
\hline & Temp: $(\mathrm{T})$ & -0.62752 & 0.053183 & 0 \\
\hline & Color X anchor (WS) & 26.86182 & 8.24157 & 0.0011 \\
\hline & Color X Temp (WT) & 0.53587 & 0.056895 & 0 \\
\hline & Anchor X Temp (ST) & -0.30828 & 0.091416 & 0.0008 \\
\hline & $\begin{array}{l}\text { Color X Anchor X Temp } \\
\text { (WST) }\end{array}$ & -0.32339 & 0.103103 & 0.0017 \\
\hline Residual & & & 0.07194 & \\
\hline
\end{tabular}

Slope coefficient for each color*anchor combination is given in Table 6. The interaction plots for delta, beta and kappa, shown in Fig 2, are constructed using the slopes in Table 6. The pairwise tests of equality slopes are specified in Table 7. 
Table 6. Estimates of Color*Anchor Interaction Ta Slopes for All Parameter Effect Milk PM Activity

\begin{tabular}{|l||l|l|l|}
\hline $\begin{array}{l}\text { Parameter/ } \\
\text { Treatments }\end{array}$ & Color & Anchor & Slopes* \\
\hline Delta & Black & Long & 0.28514 \\
\cline { 2 - 4 } & Black & Short & -0.11841 \\
\cline { 2 - 4 } & White & Long & 0.33516 \\
\cline { 2 - 4 } & White & Short & -0.21968 \\
\hline \multirow{3}{*}{ Beta } & Black & Long & -0.37080 \\
\cline { 2 - 4 } & Black & Short & -0.40985 \\
\hline & White & Long & -0.41304 \\
\hline & White & Short & -0.19133 \\
\hline Kappa & Black & Long & 0.38436 \\
\hline & Black & Short & -0.91063 \\
\hline & White & Long & 0.13996 \\
\hline & White & Short & -0.70189 \\
\hline & *: P-value for all slope coefficients is significant \\
\hline
\end{tabular}

Table 7. Tests for Equality of Ta Slopes for All Parameter Effects: Milk PM Activity

\begin{tabular}{|l|l|l|l|l|}
\hline Parameter & $\begin{array}{l}\text { Treatment } \\
\text { Combinations }\end{array}$ & Estimates & $\begin{array}{l}\text { Std. } \\
\text { Errors }\end{array}$ & $\begin{array}{l}\text { P- } \\
\text { values }\end{array}$ \\
\hline \multirow{5}{*}{ Delta } & BS-BL & -0.40314 & 0.0270 & 0.000 \\
\cline { 2 - 5 } & WL-BL & 0.05022 & 0.067277 & 0.4555 \\
\cline { 2 - 5 } & WS-BL & -0.50385 & 0.023482 & 0.000 \\
\cline { 2 - 5 } & WL-BS & 0.45357 & 0.068055 & 0.000 \\
\cline { 2 - 5 } & WS-BS & -0.10128 & 0.025669 & 0.0001 \\
\cline { 2 - 5 } & WS-WL & -0.55484 & 0.0667 & 0.000 \\
\hline \multirow{5}{*}{ Beta } & BS-BL & -0.04204 & 0.094126 & 0.6552 \\
\cline { 2 - 5 } & WL-BL & -0.04224 & 0.084141 & 0.6262 \\
\cline { 2 - 5 } & WS-BL & 0.17710 & 0.05714 & 0.000 \\
\cline { 2 - 5 } & WL-BS & -0.00318 & 0.1126 & 0.9775 \\
\cline { 2 - 5 } & WS-BS & 0.21853 & 0.094445 & 0.0208 \\
\cline { 2 - 5 } & WS-WL & 0.22171 & 0.084123 & 0.0085 \\
\hline \multirow{5}{*}{ Kappa } & BS-BL & -1.29782 & 0.063233 & 0.000 \\
\cline { 2 - 5 } & WL-BL & -0.25804 & 0.075987 & 0.000 \\
\cline { 2 - 5 } & WS-BL & -1.08899 & 0.088738 & 0.000 \\
\cline { 2 - 5 } & WL-BS & 1.05059 & 0.056476 & 0.0314 \\
\cline { 2 - 5 } & WS-BS & 0.2084 & & \\
\cline { 2 - 5 } & WS-WL & -0.84185 & & \\
\hline
\end{tabular}

Tables $6 \& 7$, we see that delta, the minimum body temperature slope, is significantly different for both black and white animals when anchor length is short ( $p$-value $=0.00$ ), but there is no significant difference between slopes when anchor length is long ( $p$-value $=0.4555)$. We also see that the black animals have higher minimum body temperature slope (slope $=-0.11841$ ) compared to white animals (slopes $=-0.21968$ ) when measuring instrument is short. 
Fig 2. Plots of Ta Slopes by Color*Anchor Interaction for Delta (Minimum Body Temperature), Beta (Range) and Kappa (Rate Constant): Milk pm Activity
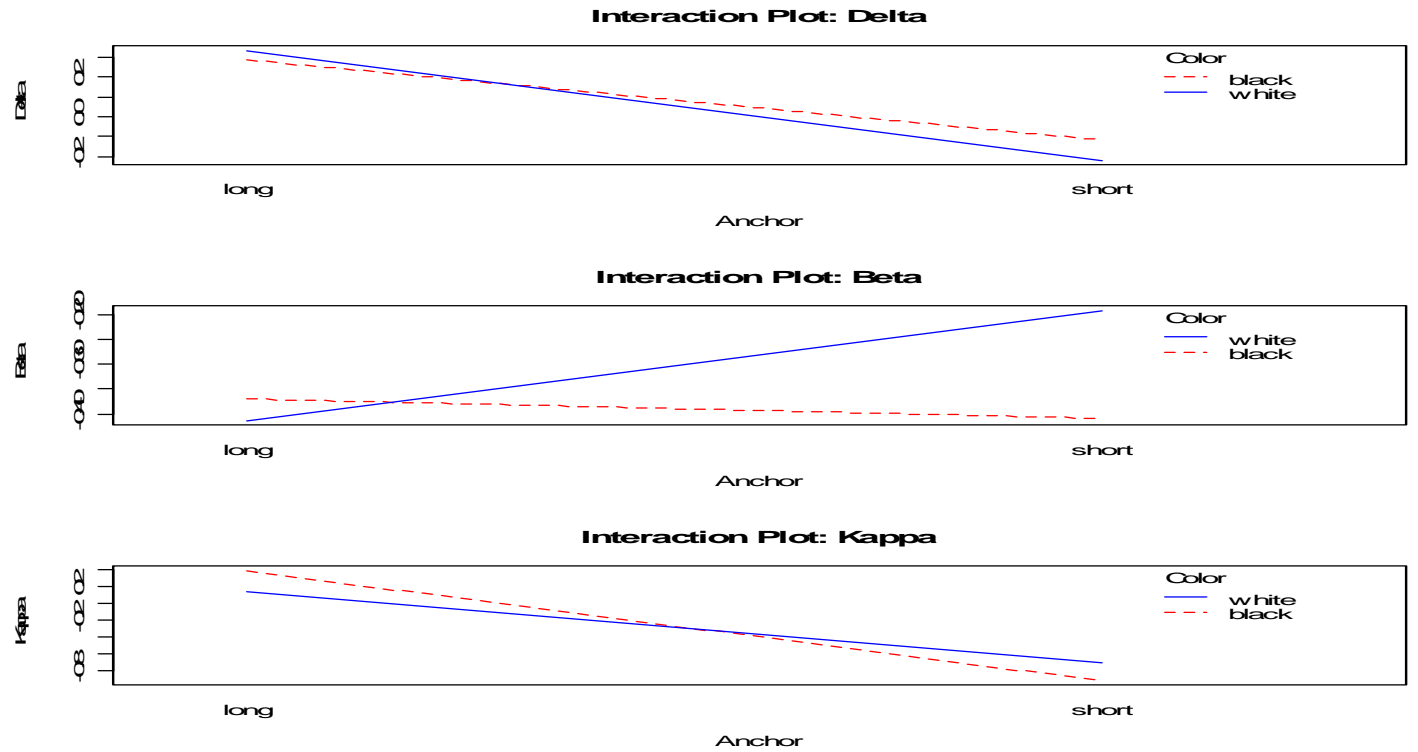

From Fig 2., the rate constant slope, kappa, is significantly different for both black and white animals at each anchor length, long or short. Black animals have higher rate constant slope (slope $=0.38436$ ) when measuring instrument is long and lower rate constant slope (slope $=-0.91063$ ) when measuring is short.

For Beta, the range slope, is significantly different for both black and white animals when anchor length is short ( $p$-value $=0.0208$ ), but there is no significant difference ( $p$ value $=0.6262$ ) in slopes when anchor length is long. We also found that there is no significant differences in measuring instruments when animal coat color is black (pvalue $=0.6552$ ). White animals have higher range slope (slope $=-0.19133$ ) if measuring instrument is short compared to black animals (slope $=-0.40985$ ). The $95 \%$ confidence intervals (Table 8) provide upper and lower estimates for the fixed-effects of hair coat color and anchor length on each parameter.

Table 8. The 95\% Fixed-effects Confidence Intervals for Delta (Minimum Body Temperature), Beta (Range) and Kappa (Rate Constant): Milk PM Activity

\begin{tabular}{|l|l|l|l|l|}
\hline Parameter & Fixed Effects & Lower & Estimate & Upper \\
\hline \multirow{5}{*}{ Delta } & Intercept & 107.6044 & 108.9259 & 110.2475 \\
\cline { 2 - 5 } & Color: (W) & 26.59316 & 39.73241 & 52.87165 \\
\cline { 2 - 5 } & Anchor: (S) & -1.0669 & 1.883775 & 4.834482 \\
\cline { 2 - 5 } & Temp: (T) & -0.1055 & -0.08942 & -0.07334 \\
\cline { 2 - 5 } & Color X anchor (WS) & -46.6912 & -33.1423 & -19.5934 \\
\cline { 2 - 5 } & Color X Temp (WT) & -0.72597 & -0.54848 & -0.37098 \\
\cline { 2 - 5 } & Anchor X Temp (ST) & -0.0605 & -0.02301 & 0.01448 \\
\cline { 2 - 5 } & Color X Anchor X & & & \\
& Temp(WST ) & 0.268011 & 0.450501 & 0.63299 \\
\hline Beta & Intercept & 10.73649 & 17.03525 & 23.33401 \\
\hline
\end{tabular}




\begin{tabular}{|l|l|l|l|l|}
\hline \multirow{5}{*}{ Color: (W) } & -70.9225 & -55.1849 & -39.4474 \\
\cline { 2 - 5 } & Anchor: (S) & 5.357964 & 18.5226 & 31.68723 \\
\cline { 2 - 5 } & Temp: (T) & -0.28164 & -0.20149 & -0.12133 \\
\cline { 2 - 5 } & Color X anchor (WS) & 17.39511 & 37.67052 & 57.94593 \\
\cline { 2 - 5 } & Color X Temp (WT) & 0.5214 & 0.731176 & 0.940953 \\
\cline { 2 - 5 } & Anchor X Temp (ST) & -0.40079 & -0.23282 & -0.06484 \\
\cline { 2 - 5 } & Color X Anchor X & & & \\
& Temp(WST) & -0.77298 & -0.50716 & -0.24134 \\
\hline Kappa & Intercept & 43.06529 & 51.30672 & 59.54815 \\
\cline { 2 - 5 } & Color: (W) & -52.6028 & -43.7246 & -34.8465 \\
\cline { 2 - 5 } & Anchor: (S) & 9.351162 & 23.56734 & 37.78352 \\
\cline { 2 - 5 } & Temp: (T) & -0.73122 & -0.62752 & -0.52382 \\
\cline { 2 - 5 } & Color X anchor (WS) & 10.79175 & 26.86182 & 42.93189 \\
\cline { 2 - 5 } & Color X Temp (WT) & 0.424934 & 0.535872 & 0.646811 \\
\cline { 2 - 5 } & Anchor X Temp (ST) & -0.48653 & -0.30828 & -0.13003 \\
\cline { 2 - 5 } & Color X Anchor X Temp & & & \\
& (WST) & -0.52443 & -0.32339 & -0.12235 \\
\hline
\end{tabular}

\section{3. iv. Diagnostics}

We discuss the diagnostics for Model 10. The assumption of normality for the withinevents residuals appears reasonable as evidenced by the Shapiro-Wilks test (p-value = 0.834 ) and normal probability plots, Figure 3.

Fig 3. Normal Probability Plots by Treatment Combinations

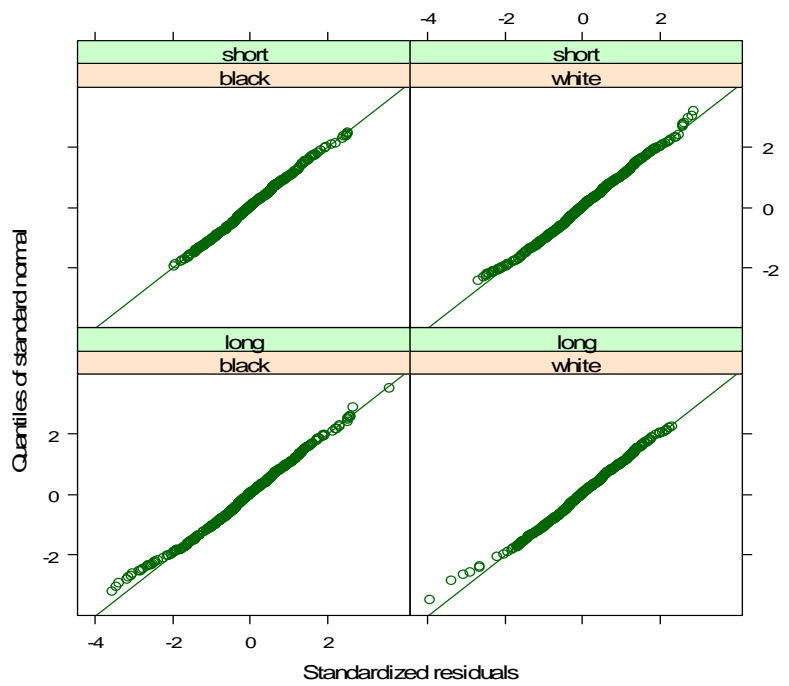

Fig 4. Box Plot of Residuals by Event

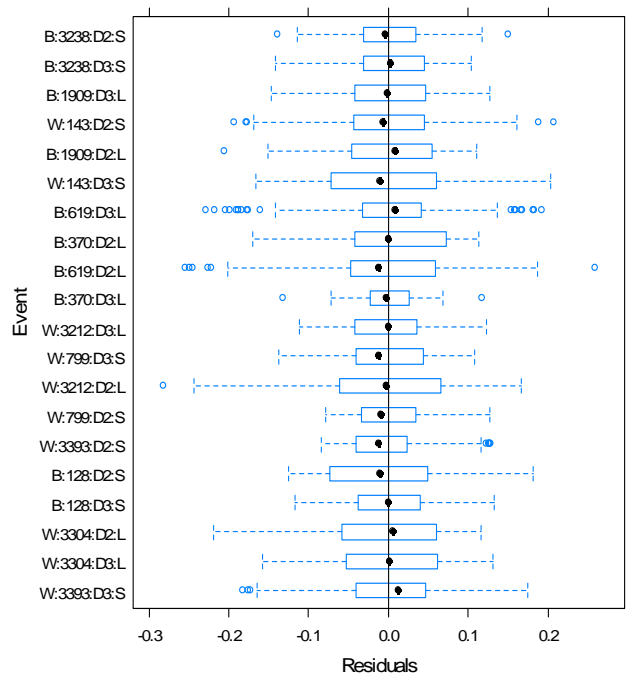

For the random-effects, the assumptions of zero expectation and independence among parameters are realistic. From the box-plots in Fig 4, we see the standardized residuals are centered at zero and there is no visible pattern among events. The bi-parameter scatter plots in Fig 5 show no relationship among parameters suggesting the parameters are not highly correlated and random-effects covariance structure is not over parameterized. 
Fig 5. Scatter Plot of Random-effects for each Parameter Combination
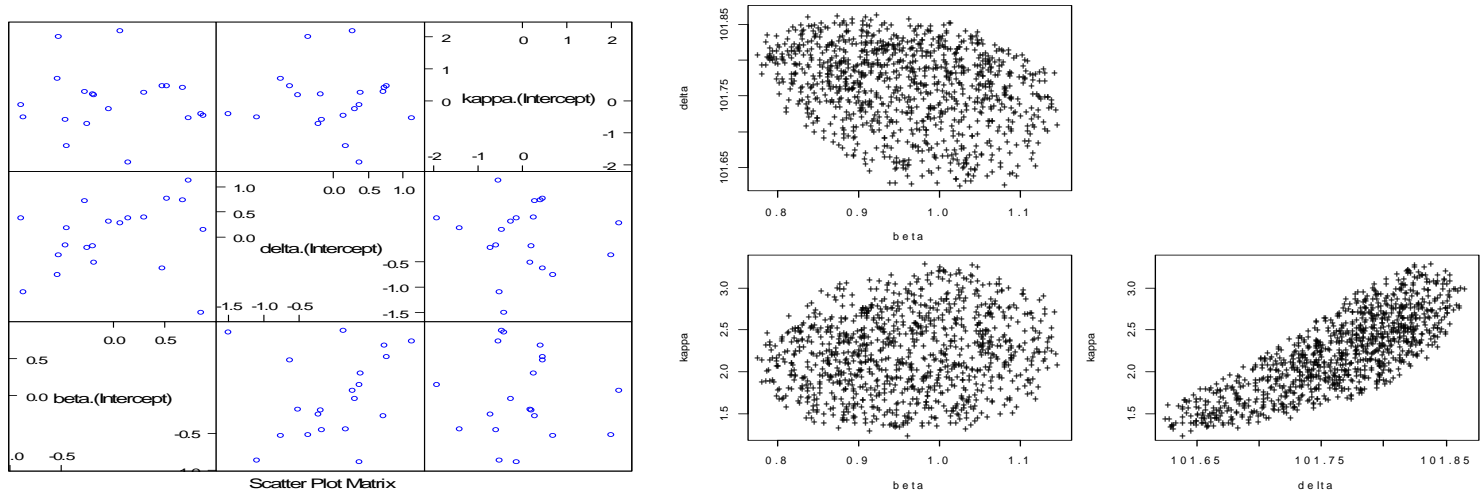

Examination of nonlinear behavior implies the planar assumption holds since the intrinsic curvature is $(\mathrm{IN}=0.317512)$. The uniform assumption is problematic. The parameter-effects curvature $(\mathrm{PE}=2.242646)$ is much larger than 0.4 but examination of the parameter estimates have indicates small bias, close to minimum variance and distributions close to normal (Table 9). From Fig 6, Parameter contour plots shows elliptical relationships between delta-beta, beta-kappa, although delta-kappa is slightly skewed. Parameter estimates have close-to-linear behavior.

Table 9: Parameter Effects for Close-to Linear-Behavior Diagnostics
\begin{tabular}{|l|l|l|l|l|}
\hline Parameter & ESTIMATE & $\%$ BIAS & $\%$ LAMBDA & SKEWNESS \\
\hline Delta & 101.7661 & -0.006939 & 5.286971 & -0.636410 \\
\hline Beta & 0.961885 & 1.0481036 & 4.219828 & 0.02233003 \\
\hline Kappa & 2.173633 & 0.8523070 & 2.190161 & 0.26527093 \\
\hline
\end{tabular}

The quality of fit is illustrated in the augmented predictions plot, Fig 7, Model 10 provides good fit to the data. Population predictions under-estimate $\mathrm{Tb}$ for animal 3238 and over-estimate for animal 128. However, the event specific $\mathrm{Tb}$ predictions are close to the observed body temperature. 
Fig 7. Population predictions (fixed), Within-event predictions and Body Temperature versus Time in hours

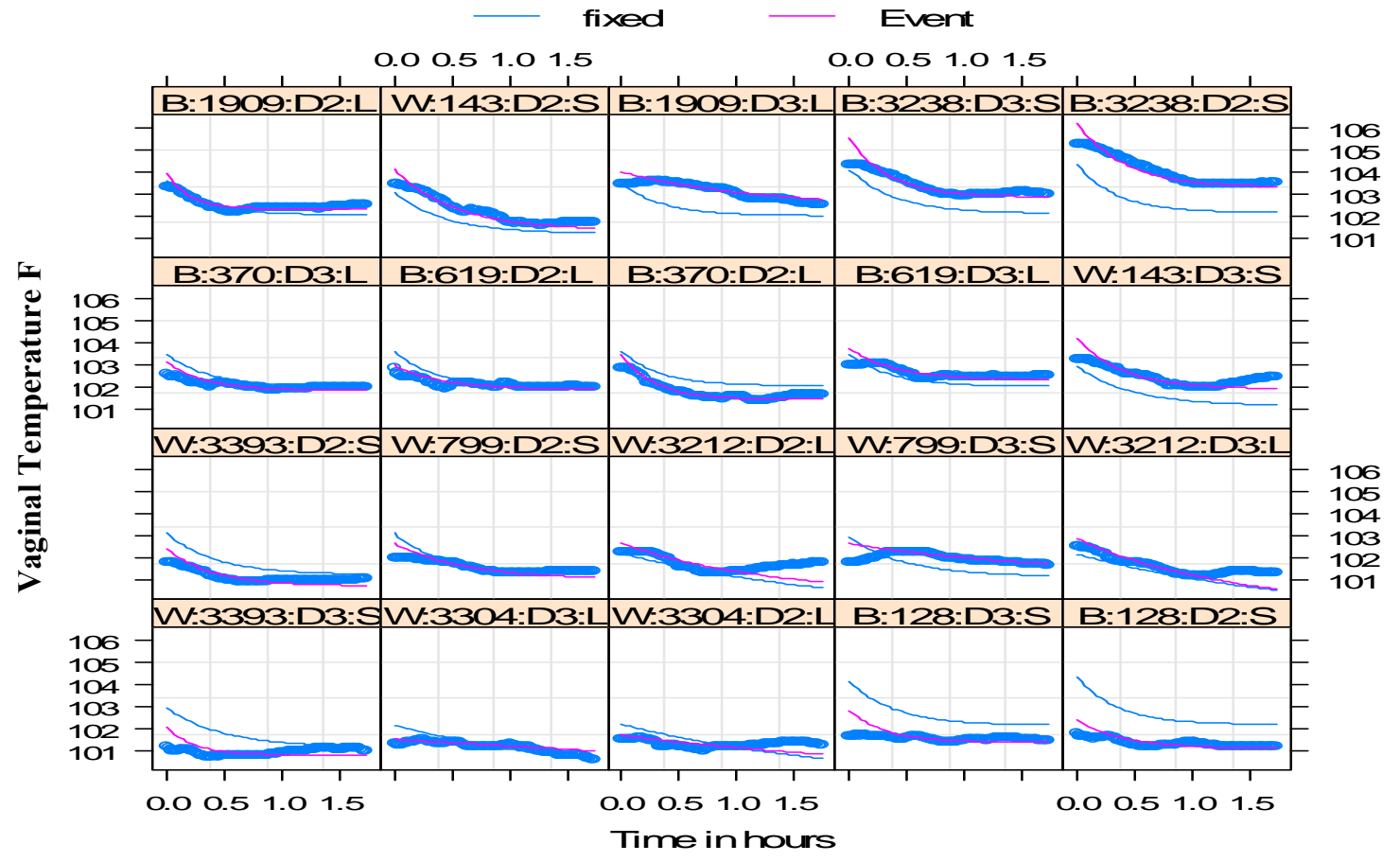

\section{Conclusions}

The assumption that during afternoon milking the rate of change in an animal's body temperature is proportional to the distance from baseline (minimum) is plausible. The resulting asymptotic exponential decay equation allows us to model body temperature over time and provides estimates for the minimum body temperature, range and rate constant parameters. The model improves when the data are grouped into cow-day events and random effects are used to account for the random variation in crossed effects. Inclusion of an environmental variable as a covariate significantly reduces variation in the model. The single most advantageous environmental variable is air temperature. In addition the inclusion of hair coat color and anchor length treatment effects further improved the model. The relationship between body and air temperature depends on both coat color and anchor length. There is a significant three-way interaction between color anchor length and air temperature for all parameters: minimum body temperature, range, and rate constant at 5\% of alpha level. Black animals have higher range, higher rate constant and lower minimum temperature slopes when the length of the measuring instrument, anchor, is long. When anchor is short, white animals have higher range, higher rate constant and lower minimum temperature slopes. For this afternoon milking data, the asymptotic regression for the $2 \times 2$ (hair color - anchor length) factorial with $\mathrm{Ta}$ covariate and crossed random effects is the model of choice.

\section{Summary}

A nonlinear exponential model with an environmental covariate is used to characterize how the body temperature changes over time for afternoon milking of Holstein Cows. 
The productivity of Holstein cows is associated with physiological and environmental conditions. There are many physiological changes, but in this study we focused only on the difference in hair coat color, and examine the effect of measuring device, anchor length. Unique environmental features were present since the data were collected at Mt. Kohala in Hawaii. Kohala is considered to have the highest solar radiation, highest air temperature, and highest wind speed readings in Hawaii. The environmental variables recorded were: air temperature, thermal heat index (THI), relative humidity, solar radiation, and wind speed. Data was analyzed as a complete randomized design with repeated measures over time and an environmental covariate with 2 x 2 factorial treatment designs. A nonlinear mixed model analysis with crossed random effects was used for the three-parameter asymptotic exponential decay, $\mathrm{TB}=\mathrm{delta}+\exp$ (betakappa*Time). The mixed model regression showed improvement over the fixed version. Although each environmental variable showed significant improvement when included in the model, air temperature was slightly better than THI according the information criteria. The treatment factors, hair coat color and anchor, significantly improved the fit when included in the model. The parameters for the asymptotic exponential decay model have a natural interpretation useful for describing changes in body temperature over time. The parameters depict baseline body temperature, the range (difference in initial and baseline body temperature), and the rate constant. For each parameter, there was a significant three-way interaction between color*anchor length*air temperature. When investigating hair coat color and anchor effects under the conditions of the motivating experiment, we recommend using the nonlinear exponential decay mixed model with random-effect (beta, delta and kappa) and environmental effect to analyze the data.

\section{References}

Baek, K.S., C.N. Lee, and B.S. Jeon. 2001. Effect of hair coat color of Holstein cow and type of fans on heat stress in dairy in Hawaii. Korean Soc. of Animal Production Proceedings,Seoul,Korea.

Bates, D.M, and Watts, D.G. (1980) Relative curvature measures of nonlinearity. $J . R$. Statist. Soc., 42, 1-25.

Bates, D.M. and D.G. Watts, 1988. Nonlinear Regression Analysis and its Applications. NewYork: John Wiley.

Gebremedhin, K.G., P.E. Hillman, C.N. Lee and R.J. Collier. 2007. Sweating rates dairy cows under shade and sunny environments. ASAE Proc. ASABE Annual Meeting, MN. June 2007 Pub.\# 074083]

Godfrey. R.W. and P.J. Hansen. 1996. Reproduction and milk yield of Holstein cows in the Virgin Islands as influence by time of the year and hair coat. Ach. Latinoam. Prod. Anim. 4(1)31-44.

Hillman, P.E., C.N. Lee, J.R. Carpenter, K.S. Baek and A. Parkhurst. 2001. Impact of hair coat color on thermoregulation of dairy cows to direct sunlight. ASAE Annual 
Meeting, paper \# 014031.

Huang Q., A. M. Parkhurst, T.M. Brown-Brandl, R.A. Eigenberg, and J.A. Nienaber, 2006. Evaluating Linear and Nonlinear Models for the Respiration Rate of Four Breeds of Heat Stressed Feedlot Heifers. Proceedings of Eighteenth Annual Kansas State University Conference on Applied Statistics in Agriculture Proceedings. 242-258 http://www.k-state.edu/stats/agstat.conference/2006

Lee, C.N., KS Baek and A. Parkhurst. 2003. Hair coat color may influence longevity of Holstein cattle in the tropics. J.Dairy Sci. 86 [Suppl. 1]p.17.

Pinheiro, J.C. and D.M. Bates. 2000. Mixed-Effects Models in S and S-PLUS. New York: Springer-Verlag.

R Development Core Team. 2004. R: A Language and Environment for Statistical Computing. R Foundation for Statistical Computing, Vienna, Austria. ISBN 3-90005107-0, URL http://www.R-project.org.

Schabenberger, O., F. J. Pierce, 2002. Contemporary statistical models for the plant and soil sciences, Boca Raton, Fla.: CRC Press

Zhou M., A.M. Parkhurst, R.A.Eigenberg, J.Nienaber, L.Hahn, 2006. Evaluating Nonlinear Crossed Random Effects Models for Comparing Temperature of Eating Pigs Under Different Thermal Environments. Proceedings of Eighteenth Annual Kansas State University Conference on Applied Statistics in Agriculture Proceedings. 180-200 http://www.k-state.edu/stats/agstat.conference/2006

Zhou M., A.M. Parkhurst, B.C. Pollard, R.J. Collier, 2007. Using a Nonlinear Crossed Random Effects Model with Three-Way Treatment Structure for Describing Circadian Patterns of Serum Prolactin Concentrations in Heat Stressed Holsteins. Proceedings of Nineteenth Annual Kansas State University Conference on Applied Statistics in Agriculture Proceedings. 126-143 http://www.k-state.edu/stats/agstat.conference/2007

APPENDIX: The NLME code for fitting the nonlinear mixed-effects model formulated by Eq. 1, Eq. 2 and Eq. 3 with and without covariate and treatments and assuming diagonal B and independent errors

mlkpm<-nlme(temp beta*exp(-kappa*NewTimes)+delta, fixed $=$ beta + delta + kappa $\sim 1$, random=pdDiag $($ beta + delta + kappa $\sim 1)$, data $=$ mlkpm.evt.gp, $\operatorname{start}=\mathrm{c}(1.009577696,101.97459800,1.9831498334))$

mlkpm.AirT<-update $(\mathrm{mlkpm}$,fixed=beta+delta + kappa $\sim \mathrm{ITempF}, \mathrm{start}=\mathrm{c}(1.23009577696,0,102.97459800,0,2.04554,0))$ mlkpm.trt.nlme<- update(cows.mlkpm.AirT, fixed=beta + delta + kappa $\sim$ factor(color)*factor(anchor)*ITempF, start $=\mathrm{c}(1.35906686,0.27263037,0.10209991,-0.46817895,1.23009577696,0.3456,1.23009577696$, $110.34156146,3.98632500,-4.05236278,-2.81654368,1.39583892,0.21361216,102.974,0,0.12257645$, $0.17505570,-0.11010093,-0.06619657,0.05504730,0,2.4567,0,2.3456))$ 